\title{
ON THE STAR FOREST POLYTOPE FOR TREES AND CYCLES
}

\author{
Meziane Aider $^{1}$, Lamia Aoudia ${ }^{1}$, Mourad Bä̈OU $^{2}$, \\ A. Ridha Mahjoub ${ }^{3}$ and Viet Hung Nguyen ${ }^{4, *}$
}

\begin{abstract}
Let $G=(V, E)$ be an undirected graph where the edges in $E$ have non-negative weights. A star in $G$ is either a single node of $G$ or a subgraph of $G$ where all the edges share one common end-node. A star forest is a collection of vertex-disjoint stars in $G$. The weight of a star forest is the sum of the weights of its edges. This paper deals with the problem of finding a Maximum Weight Spanning Star Forest (MWSFP) in $G$. This problem is NP-hard but can be solved in polynomial time when $G$ is a cactus [Nguyen, Discrete Math. Algorithms App. 7 (2015) 1550018]. In this paper, we present a polyhedral investigation of the MWSFP. More precisely, we study the facial structure of the star forest polytope, denoted by $\operatorname{SFP}(G)$, which is the convex hull of the incidence vectors of the star forests of $G$. First, we prove several basic properties of $\operatorname{SFP}(G)$ and propose an integer programming formulation for MWSFP. Then, we give a class of facet-defining inequalities, called $M$-tree inequalities, for $\operatorname{SFP}(G)$. We show that for the case when $G$ is a tree, the $M$-tree and the nonnegativity inequalities give a complete characterization of $\operatorname{SFP}(G)$. Finally, based on the description of the dominating set polytope on cycles given by Bouchakour et al. [Eur. J. Combin. 29 (2008) 652-661], we give a complete linear description of $\operatorname{SFP}(G)$ when $G$ is a cycle.
\end{abstract}

Mathematics Subject Classification. 90C27, 90C10, 05Cxx.

Received June 1, 2015. Accepted September 4, 2018.

\section{INTRODUCTION}

Given an undirected graph $G=(V, E)$ where $n=|V|$ and $m=|E|$, a star in $G$ is either a single node of $G$ or a subgraph of $G$ where every edge shares one common end-node. The latter is called the center of the star when the star is not reduced to a single node. If the star is a single edge, then any of its end-nodes can be designated as the center. A star forest is a collection of vertex-disjoint stars in $G$. An edge dominating set in $G$ is an edge subset $F \subseteq E$ such that for any edge $e$ in $G$ either $e \in F$ or $e$ shares at least one common end-node with some edge in $F$. A dominating set in $G$ is a node subset $S \subseteq V$ such that for any node $u \in V$ either $u \in S$ or $u$ is neighbor with some node in $S$. We suppose that the edges in $G$ have non-negative weights (note that

Keywords. Combinatorial optimization, polyhedral combinatorics, star forest, facility location, dominating set.

1 Laromad, Faculty of Mathematics, University of Sciences and Technology, Houari Boumediéne, Algeirs, Algeria.

2 LIMOS, CNRS UMR 6158, Clermont-Ferrand, France.

3 Université Paris-Dauphine, PSL, CNRS UMR 7243 LAMSADE, Place du Maréchal De Lattre de Tassigny, 75775, Paris Cedex 16, France.

4 Sorbonne Université, CNRS UMR 7606, LIP6 - 4 place Jussieu, Paris, France.

* Corresponding author: Hung. Nguyen@lip6.fr 

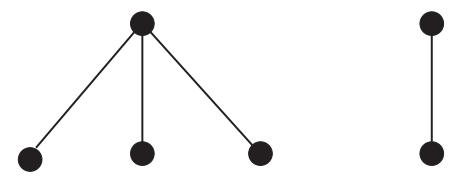

Figure 1. A star forest of weight 4 with weights 1 on the edges.

the unweighted case can be seen as a special weighted case when weights are 0 or 1), then the weight of a star forest or an edge dominating set is the sum of the weights of its edges. The Maximum Weight spanning Star Forest Problem (MWSFP) is to find a star forest spanning the nodes of $G$ of maximum weight. The Minimum Weight Edge Dominating Problem (MWEDP) is to find an edge dominating set in $G$ of minimum weight. If the nodes are weighted, the weight of a dominating set is the sum of the weights of its nodes. The Minimum Weight Dominating Set Problem (MWDSP) is to find a minimum weight dominating set in $G$. The two last problems are well-known to be $N P$-hard. They have been the subject of many works in the literature [20], [13]. The MWSFP, however, is a recent problem which has been introduced by Nguyen et al. in [17]. It has applications in several areas, especially in computational biology [17] and automobile industry [1]. In [17], the authors show the $N P$-hardness of MWSFP by observing that in a maximal star forest $F$ (a maximal star forest is a star forest to which no more edge can be added), the set of the centers of the stars in $F$ is a dominating set of $G$. Conversely, for any dominating set $S$ we can build a maximal star forest with centers as the nodes belonging to $S$. Thus, given a maximal star forest $F$, there exists a dominating set $S$ such that $|S|=|V|-|F|$ and vice versa. Hence the case of $0 / 1$ weights of the MWSFP is $N P$-hard by a reduction from the of $0 / 1$ weights case of the MWDP. In [17], the authors also give a linear time algorithm to solve the MWSFP when $G$ is a tree and a $\frac{1}{2}$-approximation algorithm for the general case. Since then, the MWSFP has been intensively investigated, in particular for the unweighted version. Nguyen et al. [17] prove that the problem is APX-hard by presenting an explicit inapproximability bound of 259/260, and present a combinatorial 0.6-approximation algorithm for the unweighted MWSFP. Polynomial-time algorithms are presented for special classes of graphs such as planar graphs and trees in the same paper. Chen et al. [12] present a better approximation algorithm with ratio 0.71 for unweighted MWSFP. Later, Athanassopoulos et al. [2] improve this approximation ratio to 0.803 by using the fact that the problem is a special case of the complementary set cover problem. Interesting generalizations including node-weighted and edge-weighted versions of the MWSFP have also been considered. In $[12,17]$ the authors present approximation algorithms and APX-hardness results for these problems as well. Stronger inapproximability results for these problems recently appeared in [11,14]. For the weighted version, Nguyen [18] has given a linear time algorithm for solving the MWSFP when $G$ is a cactus.

Let $\operatorname{SFP}(G)$ (respectively $\operatorname{EDP}(G)$ ) be the convex hull of the incidence vectors of the star forests (respectively the edge dominating sets) in $G$. Let $\mathbb{R}^{n}$ be the real space indexed by the nodes in $V$. Let $D$ be any dominating set in $G$, let $\chi(D) \in \mathbb{R}^{n}$ be the incidence vector of $D$, defined as

$$
\chi(D)_{v}=\left\{\begin{array}{l}
1 \text { if } v \text { is a nodes in } V \text { and } v \in D \\
0 \text { otherwise. }
\end{array}\right.
$$

Let $D P(G)$ be the convex hull of the incidence vectors of the dominating sets in $G$. To the best of our knowledge, no polyhedral investigation has been done for $\operatorname{SFP}(G)$ and $\operatorname{EDP}(G)$ though some integer formulations have been used in approximation algorithms for the MWSFP and the MWEDP. There are, however, several works on $D P(G)$, in particular, Saxena [19] has given a complete description for $D P(G)$ when $G$ is a tree, and Bouchakhour et al. [8] have given a complete description for $D P(G)$ when $G$ is a cycle. In [16], Mahjoub has given a complete description of $D P(G)$ in threshold graphs. And in [9], Bouchakour and Mahjoub have studied compositions for the polytope $D P(G)$ in graphs that decompose by one-node cutsets. 
In this paper, we present a polyhedral investigation of the MWSFP for trees and cycles. More precisely, we study the facial structure of the star forest polytope. In the first part of the paper, we give a complete characterization of $\operatorname{SFP}(G)$ when $G$ is a tree, which is obtained by projection of a simple extended formulation issued from the work of Baïou and Barahona [3] on the uncapacitated facility location polytope. Also, we show that the facet-defining inequalities for $\operatorname{SFP}(G)$ when $G$ is a tree can be generalized to valid inequalities for $\operatorname{SFP}(G)$ when $G$ is a arbitrary graph. These inequalities define facets for $\operatorname{SFP}(G)$ under certain conditions, and can be separated in polynomial time.

In the second part of the paper, we give a complete description for $\operatorname{SFP}(G)$ when $G$ is a simple cycle $C$. More precisely, we establish the relation between spanning star forests and dominating sets when the graph is a simple cycle $C$ and give a complete linear description for $S F P(C)$ based on the one for $D P(C)$ given by Bouchakhour et al. [8].

The paper is organized as follows. In the next section, we describe some properties of $S F P(G)$ and give an integer programming formulation for the MWSFP. In section 3, we introduce a class of valid inequalities, called the $M$-tree inequalities, for $\operatorname{SFP}(G)$ and give a complete description for $\operatorname{SFP}(G)$ when $G$ is a tree. In Section 4, we present a complete description for $\operatorname{SFP}(G)$ when $G$ is a cycle.

In the rest of this section, we give some notations that will be used in the paper. For $x \in \mathbb{R}^{m}$, given any $F \subseteq E$, we let $x(F)$ denote $\sum_{e \in F} x_{e}$. For $x \in \mathbb{R}^{n}$, given a set $S \subseteq V$, we let $x(S)$ denote $\sum_{v \in S} x_{v}$. Given a set of vertices $S$, we denote by $E(S)$ the set of edges with both ends belonging to $S$. Let $v \in V$, the neighborhood of $v$, denoted by $N(v)$, is the vertex set consisting of $v$ and the nodes which are adjacent to $v$. Given any edge subset $F \subseteq E$, let $V(F)$ denote the set of the end-nodes of the edges in $F$. We call a 3-path a simple path having 3 edges in $G$ and a 3 -cycle a triangle in $G$. Let $\mathcal{P}_{4}$ (respectively $\mathcal{C}_{3}$ ) denote the collection of the 3-paths (resp. 3-cycles) in $G$.

\section{BAsic PROPERTIES OF $S F P(G)$ AND INTEGER PROGRAMMing FORMULATion FOR THE MWSP}

\subsection{Basic properties of $\operatorname{SFP}(G)$.}

The following remark is about zero vector $\mathbf{0} \in \mathbb{R}^{m}$ which is the incidence vector associated with the single node star forests.

Remark 2.1. The zero vector $\mathbf{0} \in \mathbb{R}^{m}$ is an extreme point of $S F P(G)$.

Proof. We can see that $\mathbf{0}$ is the incidence vector associated with the single node star forests and as for any $x \in \operatorname{SFP}(G)$ and any $e \in E, x_{e} \geq 0, \mathbf{0}$ is an extreme point of $\operatorname{SFP}(G)$.

Hence, $\operatorname{SFP}(G)$ is a polytope pointed at $\mathbf{0}$. Moreover, the following theorem shows that $S F P(G)$ is full dimensional.

Theorem 2.2. $\operatorname{SFP}(G)$ is a full dimensional polytope, i.e. $\operatorname{dim}(\operatorname{SFP}(G))=m$.

Proof. Suppose that the incidence vectors of all the star forests in $G$ satisfy some equality $\alpha^{t} x=\beta$. As $\alpha^{t} \mathbf{0}=\beta$, $\beta=0$. As any edge $e \in E$ is a star forest in $G$, we have $\alpha_{e}=\beta=0$ for all $e \in E$.

Theorem 2.3. All the facet-defining inequalities of $S F P(G)$, that are different from $x_{e} \geq 0$ for some $e \in E$, are of the form $a^{t} x \leq b$ with $a \in \mathbb{R}_{+}^{m}$ and $b \geq 0$ scalar.

Proof. Let $a^{t} x \leq b$ be any facet-defining inequality for $\operatorname{SFP}(G)$ which is not $x_{e} \geq 0$ for some $e \in E$. As $a^{t} \mathbf{0} \leq b$, we have $b \geq 0$. Suppose that for some edge $e, a_{e}<0$. As $a^{t} x \leq b$ defines a facet different from $x_{e} \geq 0$, there exists a star forests $F$ in $G$ containing $e$ such that $a^{t} \chi^{F}=b$. As $F^{\prime}=F \backslash\{e\}$ is also a star forest, we have $a^{t} \chi^{F^{\prime}}=a^{t} \chi^{F}-a_{e}=b-a_{e}>b$. This contradicts the fact that $a^{t} x \leq b$ is valid for $\operatorname{SFP}(G)$. 
Given $a^{t} x \leq b$ any facet-defining inequality of $S F P(G)$, the support graph of $a^{t} x \leq b$ is the subgraph $G_{a}=\left(V_{a}, E_{a}\right)$ of $G$ induced by the edges $e \in E$ such that $a_{e}>0$. A tight star forest $F$ with respect to $a^{t} x \leq b$ is a star forest with which the associated incidence vector satisfies $a^{t} x \leq b$ at equality. A star forest $F$ is maximal with respect to an edge subset $E^{\prime} \subseteq E$ if for any edge $e \in E^{\prime} \backslash F, F \cup\{e\}$ is not anymore a star forest.

Theorem 2.3 implies the following corollary.

Corollary 2.4. Given $a^{t} x \leq b$ any facet-defining inequality of $S F P(G)$ with $G_{a}=\left(V_{a}, E_{a}\right)$ its support graph, all the tight star forests with respect to $a^{t} x \leq b$ are maximal with respect to $E_{a}$.

Lemma 2.5. Let $\bar{G}=(\bar{V}, \bar{E})$ be any induced subgraph of $G$, then any facet-defining inequality for $S F P(\bar{G})$ also defines a facet for $\operatorname{SFP}(G)$.

Proof. First notice that the theorem trivially holds for the trivial inequalities $x(e) \leq 1$ for all $e \in \bar{E}$, since these inequalities define facets for $\operatorname{SFP}(G)$ for any graph $G$. Let $I$ be any facet-defining inequality for $\operatorname{SFP}(\bar{G})$, different from $x(e) \leq 1$ for all $e \in E$. Then $I$ defines a facet for $\operatorname{SFP}(G)$ if we are able to show that for any edge $i j \in E \backslash \bar{E}$, there always exists a tight star forest $F$ in $\bar{G}$ with respect to $I$ such that $F \cup\{i j\}$ is also star forest in $G$. Suppose that for some edge $i j \in E \backslash \bar{E}$, no such $F$ exists. This implies that for every tight star forest $F$ in $\bar{G}$ w.r.t $I, F \cup\{i j\}$ is not a star forest in $G$. In this case, exactly one node $i$ or $j$, say $i$, belongs to $\bar{V}$. Moreover, as $\bar{G}$ is an induced subgraph of $G$, every tight forest $F$ in $\bar{G}$ w.r.t $I$ should contain an edge $i k$ such that $i$ is of degree 1 in $F$ and $k$ is of degree at least 2 in $F$. Otherwise, $F \cup\{i j\}$ would be a star forest of $G$. Then every tight star forest $F$ in $\bar{G}$ w.r.t $I$ also satisfies $x\left(\delta_{\bar{G}}(i)\right)=1$. This, together with Theorem 2.2, implies that $I$ should be $x\left(\delta_{\bar{G}}(i)\right) \leq 1$. Since $I$ is different from $x(e) \leq 1$ for all $e \in E, i$ should be of degree at least 2 in $\bar{G}$. But then $x\left(\delta_{\bar{G}}(i)\right) \leq 1$ is not valid for $S F P(\bar{G})$, a contradiction.

\subsection{Integer programming formulation for the MWSFP}

In this subsection, we give an integer programming formulation for MWSFP. First we state the following lemma.

Lemma 2.6. A graph is a star forest iff it does not contains 3-paths and 3-cycles.

Proof. It can be immediately verified from the definition of a star forest.

Let us consider the following integer program.

\begin{tabular}{|c|c|c|}
\hline & \multicolumn{2}{|l|}{$\max c^{T} x$} \\
\hline & s.t. & \\
\hline & $x(P) \leq 2$ & for all $P \in \mathcal{P}_{4}$ \\
\hline & $x(C) \leq 2$ & for all $P \in \mathcal{C}_{3}$ \\
\hline & $0 \leq x_{e} \leq 1$ & for all $e \in E$ \\
\hline & $x$ integer & \\
\hline
\end{tabular}

Inequalities (2.1), called the 3-path inequalities, state the fact that a star forest can only take at most 2 edges in a 3-path. Similarly, inequalities (2.2), called the 3-cycle inequalities, state the fact that a star forest can only take at most 2 edges in a 3 -cycle. Inequalities (2.3) are the trivial inequalities.

Theorem 2.7. $(I P)$ is equivalent to the MWSFP.

Proof. It is clear that by inequalities (2.1) and (2.2) in a solution of $(I P)$ there is neither 3-paths and nor 3 -cycles. By Lemma 2.6, this solution represents a star forest. 


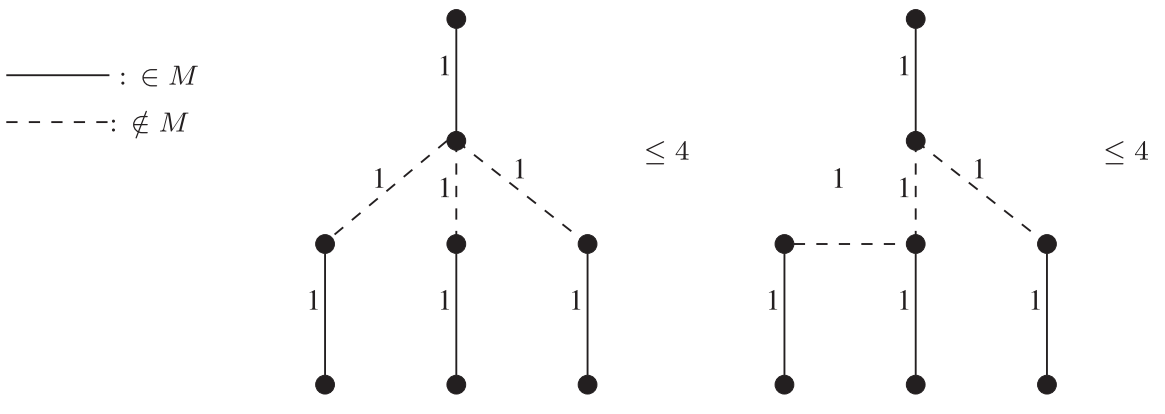

Figure 2. A $M$-tree inequalities associated with a $M$-matching $M$ of cardinality 4 .

\section{3. $M$-Tree inequalities AND COMPlete Description of $\mathrm{SFP}(\mathrm{G})$ in TREeS}

In this section, we shall give a complete description of $\operatorname{SFP}(G)$ in trees. For this, let us first describe a class of inequalities which are valid for any graph, not only for trees.

\section{1. $M$-tree inequalities}

In this subsection, $G$ is an arbitrary graph (not necessarily a tree).

Definition 3.1 ( $M$-tree). A $M$-tree $\tau$ is a tree in which every non-pendant node is connected to exactly one pendant node (leaf).

Given a $M$-tree $\tau$, let us call $M$-matching of $\tau$, the set of the edges incident to the leaves of $\tau$.

Definition 3.2 ( $M$-tree inequality). The $M$-tree inequality associated with a $M$-tree $\tau$ is the inequality $x(\tau) \leq$ $|M|$ where $M$ is the $M$-matching of $\tau$.

We can remark that the $M$-tree inequalities generalize inequalities $x_{e} \leq 1$ for $e \in E$ and the 3-path inequalities. These are $M$-tree inequalities with $|M|=1$ and $|M|=2$, respectively.

Theorem 3.3. The $M$-tree inequalities define facets for $\operatorname{SFP}(G)$.

Proof. Let $\tau$ be any $M$-tree and $M$ the $M$-matching of $\tau$, let us consider the corresponding $M$-tree inequality

$$
x(\tau) \leq|M| .
$$

Let us first prove the validity. Let $F$ be any star forest of $G$. We will prove the validity by showing that $|F \cap \tau| \leq|M|$. If $F$ only contains the edges in $M$, then $|F \cap \tau| \leq|M|$. If $F$ only contains edges in $\tau \backslash M$ then $|F \cap \tau| \leq|\tau \backslash M|$ and, from the definition of a $M$-tree, $|\tau \backslash M|=|M|-1<|M|$. Now suppose that $F$ contains edges in both $M$ and $\tau \backslash M$. If $F \cap \tau$ is a matching then $|F \cap \tau| \leq|M|$ since $M$ covers all the nodes of $\tau$. So suppose that $F \cap \tau$ is not a matching. Thus, $F \cap \tau$ should contain a (sub)star $S$ with, say $u_{2}$, as center, which contains at least two edges in $F$ : one edge, say $u_{1} u_{2}$, which belongs to $M$ and one other, say $u_{2} v_{2}$, which belongs to $\tau \backslash M$. As $v_{2}$ should be also covered by $M$, there exists an edge $v_{1} v_{2} \in M$. As $u_{1} u_{2}$ and $u_{2} v_{2}$ are in $F, v_{1} v_{2} \notin F$. Moreover, by definition of $M$-tree, $v_{1}$ should be of degree 1 in $\tau$. It follows that each edge in $F$ which belongs to $\tau \backslash M\left(\right.$ e.g. $\left.u_{2} v_{2}\right)$ correspond exactly to another edge $\left(e . g . v_{1} v_{2}\right)$ in $M \backslash F$. Hence, $|F \cap(\tau \backslash M)| \leq|M \backslash F|$ which implies $|(F \cap(\tau \backslash M)) \cup(F \cap M)| \leq|(M \backslash F) \cup(F \cap M)|=|M|$. As $M \subset \tau$, $|(F \cap(\tau \backslash M)) \cup(F \cap M)|=|F \cap \tau|$. Thus, $|F \cap \tau| \leq|M|$.

Let us prove now that (3.1) defines a facet for $S F P(G)$. Suppose that there exists a facet-defining inequality $\alpha^{t} x \leq \beta$ for $\operatorname{SFP}(T)$ such that all the star forests satisfying (3.1) at equality, satisfy also $\alpha^{t} x \leq \beta$ at equality. 
Since by Theorem 2.2, $\operatorname{SFP}(T)$ is full dimensional, it suffices to show that $\alpha^{t} x \leq \beta$ is a positive multiple of (3.1).

Let us remark that $M$ is a star forest satisfying (3.1) at equality and hence also statisfies $\alpha^{t} x \leq \beta$ at equality, i.e. $\alpha(M)=\beta$. For any edge $e \in E \backslash \tau$, we can see that $M \cup\{e\}$ is also star forest satisfying (3.1) at equality. Hence, $\alpha(M \cup\{e\})=\beta$. This implies that $\alpha_{e}=0$ for all $e \in E \backslash \tau$.

Let $u_{2} v_{2}$ be any edge in $\tau \backslash M$ and $u_{1} u_{2}$ and $v_{1} v_{2}$ be the edges in $M$ incident to $u_{2}$ and $v_{2}$ respectively. We can see that $M,\left(M \backslash\left\{u_{1} u_{2}\right\}\right) \cup\left\{u_{2} v_{2}\right\}$ and $\left(M \backslash\left\{v_{1} v_{2}\right\}\right) \cup\left\{u_{2} v_{2}\right\}$ are all star forests satisfying (3.1) at equality. This implies that $\alpha_{u_{1} u_{2}}=\alpha_{u_{2} v_{2}}=\alpha_{v_{1} v_{2}}$. If we extend this to all the edges in $\tau \backslash M$, we obtain that $\alpha_{e}=\alpha_{e^{\prime}}$ for all $e, e^{\prime} \in \tau$. Hence, $\alpha^{t} x \leq \beta$ is a positive multiple of (3.1), which ends the proof of the theorem.

\subsection{Complete description of $\operatorname{SFP}(G)$ in trees}

From now on and throughout this section, $G$ will be a tree denoted by $T$.

Proposition 3.4. All the maximal star forests with respect to a $M$-tree $\tau$ are of cardinality $|M|$ where $M$ is the $M$-matching of $\tau$.

Proof. Let $S$ be any maximal star forest in $\tau$. Let $m=|M|$ then $|V(\tau)|=2 m$ and $|\tau|=2 m-1$. By the validity of $M$-tree inequalities, we have $|S| \leq m$. We will prove that $|S| \geq m$ by showing that each edge in $M$ correspond to an edge in $S$. Let $v_{1}$ be any leaf in $\tau$ and let $v_{2}$ be the non-pendant node such that the edge $v_{1} v_{2} \in M$. We distinguish two cases:

- $v_{1} \notin S$. In this case, $v_{2}$ should belong to $V(S)$ since otherwise we can add $v_{1} v_{2}$ to $S$ and $S$ remains a star forest. Moreover, $v_{2}$ should be of degree 1 in $S$ since otherwise we can also add $v_{1} v_{2}$ to $S$ and $S$ remains a star forest. Thus, the edge $v_{1} v_{2}$ correspond to the edge incident to $v_{2}$ in $S$.

- $v_{1} \in S$. Then the edge $v_{1} v_{2}$ should belong to $S$ and hence it corresponds to itself.

Hence, $|S| \leq m$ and $|S| \geq m$ which implies that $|S|=m$.

We will prove the following theorem.

Theorem 3.5. The $M$-tree and nonnegativity inequalities completely define $S F P(T)$.

Proof. Suppose that $a^{t} x \leq b$ is any facet-defining inequality for $\operatorname{SFP}(T)$ which is not a $M$-tree inequality neither the nonnegativity inequality. Let $G_{a}$ be the support graph of $a^{t} x \leq b$. We can suppose without loss of generality that $G_{a}$ is a tree. Let $T_{a}$ denote this tree. Hence, $T_{a}$ is a subtree of $T$. We have two possible cases.

- $T_{a}$ is a $M$-tree. Let $F$ be any tight star forest with respect to $a^{t} x \leq b$. By Corollary $2.4, F$ is maximal with respect to $T_{a}$. Consequently, by Proposition $3.4, F$ is tight with respect to the $M$-tree inequalities. Contradiction to the fact that $a^{t} x \leq b$ is a facet defining inequality different from a $M$-tree inequality.

- $T_{a}$ is not a $M$-tree. Hence, in $T_{a}$ there must be one non-pendant node of one of the two following types:

Type 1. A non-pendant node not connected to a leaf of $T_{a}$.

Type 2. A non-pendant node connected to at least two leaves of $T_{a}$.

We distinguish two cases:

Case 1. $T_{a}$ contains only non-pendant nodes of Type 2 . In this case, one can obtain a $M$-tree $\tau$ from $T_{a}$ by keeping for each non-pendant node, only one leaf connecting to it. Let $x(\tau) \leq\left|M_{\tau}\right|$ be the $M$-tree inequality associated with $\tau$. The following remark can be easily proved.

Remark 3.6. Given a non-pendant node $v$ of Type 2, any star forest $F$ satisfying $a^{t} x \leq b$ at equality must be maximal in $\tau$ and contains either all the leaves connected to $v$ or no of them. 
Let $F$ be any tight star forest with respect to $a^{t} x \leq b$. We have $F \cap \tau$ is a maximal star forest with respect to $\tau$. Since by Proposition 3.4, every maximal star forest in $\tau$ safisfies $x(\tau) \leq\left|M_{\tau}\right|$ at equality, it follows that every star forest satisfying $a^{t} x \leq b$ at equality satisfies also the $M$-tree inequality associated with $\tau$ at equality. This contradicts the fact that $a^{t} x \leq b$ is a facet-defining inequality.

Case 2. $T_{a}$ contains at least one non-pendant node of Type 1. We will show the following lemma.

Lemma 3.7. There exists a non-pendant node s of Type 1 such that all the other non-pendant nodes of Type 1 belong to a same connected component obtained by the removal of $s$ from $T_{a}$.

Proof. We give a constructive proof.

Initialization. Let us choose any non-pendant node $s_{0}$ of Type 1 and let $i=0$.

Iteration $i$. Suppose that $T_{1}^{i}, \ldots, T_{p_{i}}^{i}$ are the subtrees of $T_{a}$ obtained if $s_{i}$ is removed from $T_{a}$ and suppose without loss of generality that $T_{1}^{i}$ is always the tree which contains $s_{0}$. We have two possible cases.

- If all the other non-pendant nodes of Type 1 belong to a same tree $T_{k_{i}}\left(1 \leq k_{i} \leq p_{i}\right)$ then $s_{i}$ is a non-pendant node of type 1 satisfying the condition stated in the lemma. STOP.

- If the other non-pendant nodes of Type 1 belong to at least two trees. Suppose without loss of generality that $T_{p_{i}}^{i}$ is one of them. Let us choose $s_{i+1}$ to be any non-pendant node of Type 1 in $T_{p_{i}}^{i}$ and set $i \leftarrow i+1$. Reiterate.

We have the following remark.

Remark 3.8. $T_{1}^{i}$ contains all the node previously chosen $s_{0}, \ldots, s_{i-1}$ which are all distincts. The set containing these nodes is called the kernel.

Thus, the procedure should be ended by finding a non-pendant node of Type 1 satisfying the condition stated in the lemma after at most $\left|V\left(T_{a}\right)\right|$ iterations since the kernel grows after each iteration.

Let $s$ be a non-pendant node of Type 1 satisfying the condition stated in Lemma 3.7. Let $T_{1}$ be the connected component which contains the other non-pendant nodes of Type 1 obtained by removing $s$ from $T_{a}$. We can observe that $s$ is connected to $T_{1}$ by only one edge and the subgraph $H$ of $T_{a}$ induced by the edges in $T_{a} \backslash T_{1}$ is a tree. Moreover, $H$ is either a $M$-tree or a tree which contains some non-pendant nodes of Type 2 but no one of Type 1. Hence, in all the cases as we have proved in Case 1, $H$ contains a $M$-tree $\tau$ which contains all the non-pendant nodes of $H$. Let $u$ be the neighbour of $s$ in $T_{1}$, i.e. the edge $s u \in H$. Observe that $s u$ is a bridge in $T_{a}$ that links $H$ to $T_{1}$. We can see that any maximal star forest in $T_{a}$, whatever it contains su or not, contains a maximal star forest in $H$ (if a maximal star forest in $T_{a}$ does not contain $s u$, it must contain some edge $s v$ where $v$ is of degree at least 2 in the star forest). The latter, by Remark 3.6, contains a maximal star forest in $\tau$. By Corollary 2.4, any star forest $F$ satisfying $a^{t} x \leq b$ at equality is maximal in $T_{a}$. Hence $F \cap \tau$ is a maximal star forest in $\tau$. By Proposition 3.4, $F \cap \tau$ satisfies the $M$-tree inequality associated with $\tau$ at equality. This contradicts the fact that $a^{t} x \leq b$ is facet-defining.

\section{Polyhedral Results on CyCles}

In this section, $G$ will be a chordless cycle $C=(V(C), E(C))$ of $n$ nodes with $V(C)=\{1,2, \ldots, n\}$ numbered clockwise, i.e. the $n$ edges in $E(C)$ will be $e_{i}=(i, i+1)$ for $i=1, \ldots, n-1$, and the edge $e_{n}=(n, 1)$. We will sometimes use $|C|$ instead of $n,|V(C)|$ or $|E(C)|$ which are all equal. The edges in $C$ are weighted by a vector $c \in \mathbb{R}^{n}$ where $c_{i}$ is the weight associated with edge $e_{i}$ for $i=1, \ldots, n$. We also consider $L(C)=\left(V^{L}, E^{L}\right)$ the line graph of $C$ where the nodes correspond to the edges of $C$, and two nodes of $L(C)$ are adjacent if the corresponding edges are adjacent in $C$. Note that $L(C)$ is also a cycle node-weighted by vector $c$. For the sake of convenience, given a node $1 \leq i \leq n$ and an integer $t>0$, let $i+t$ designate the node $i+t$ if $i+t \leq n$ and the node $(i+t) \bmod n$ if $(i+t)>n$. For two nodes $u$ and $v$ with $v=u+t$ for some integer $t>0$ in $C$, let $C(u, v)$ denote the path $(u+1, \ldots, u+t-1)$ of $C$ between $u+1$ and $u+t-1$ (note that the path does not contain 

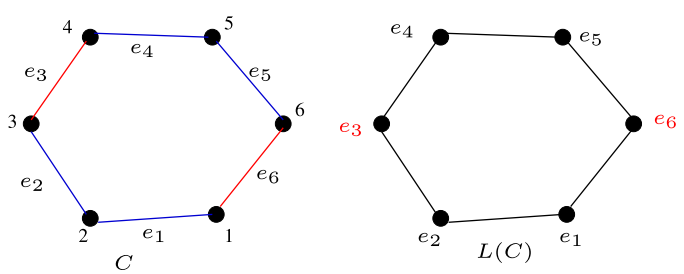

Figure 3. A cycle $C$ and its line graph $L(C)$. The edge subset $\left\{e_{1}, e_{2}, e_{4}, e_{5}\right\}$ is a star forest in $C$ and its complement $\left\{e_{3}, e_{6}\right\}$ is an edge dominating set in $C$ and a dominating set in $L(C)$.

$u$ and $v$ ). For two edges $e$ and $f$ in $C$ with $e=e_{i}$ and $f=e_{i+t}$ for some integer $t>0$, let $C(e, f)$ denote the path consisting of the edges $e_{i+1}, \ldots, e_{i+t-1}$ (note that the path does not contain neither $e_{i}$ nor $e_{i+t}$ ). In what follows, we will establish the relations between star forests, dominating sets and edge dominating sets on cycles. Then, using theses relations with the polyhedral results in [9] and [8], we will derive a complete description of $S F P(C)$. Note that in [18], a linear time algorithm for the MWSFP when $G$ is a cactus have been presented, hence the MWSFP can be solved in linear time in cycles.

\subsection{Star forests, dominating sets and edge dominating sets on cycles}

In the context of a cycle, Lemma 2.6 can be restated as follows.

Lemma 4.1. An edge subset $F \subset C$ is a star forest if and only if $F$ does not contain any 3-path.

The following lemma establishes the link between edge dominating sets and star forests in a cycle.

Lemma 4.2. The complement of a star forest $F$ in $C$ is an edge dominating set and vice versa.

Proof. ( $\Rightarrow)$ Let $F \subseteq E(C)$ be a star forest in $C$ and let $\bar{F}=C \backslash F$. Suppose that $\bar{F}$ is not an edge dominating set. Then there exists an edge $e_{i} \in F$ not adjacent to any edge in $\bar{F}$. As $C$ is a cycle, the neighbors of $e_{i}$, $e_{i-1}$ and $e_{i+1}$ do not belong to $\bar{F}$. Hence, $e_{i-1}, e_{i}, e_{i+1}$ form a path of length 3 in $F$, a contradiction with Lemma 4.1.

$(\Leftarrow)$ Let $E D \subseteq C$ be a edge dominating set in $C$. Let $F=C \backslash E D$ and suppose that $F$ is not a star forest. Then $F$ contains a 3 -path $\left(v_{1}, v_{2}, v_{3}, v_{4}\right)$. We can see that the edge $\left(v_{2}, v_{3}\right)$ is not dominated by $E D$ implying that $E D$ is not an edge dominating set, a contradiction.

By the one-to-one correspondence between the nodes of $L(C)$ and the edges of $C$, we have the following result.

Lemma 4.3. Any edge dominating set in $C$ is a dominating set in $L(C)$ and vice versa.

The following lemma reformulates these relations in polyhedral terms for the polytopes $S F P(C), E D P(C)$ and $D P(L(C))$.

Lemma 4.4. The following statements are equivalent:

(i) $\alpha^{t} y \geq \beta$ with $y \in \mathbb{R}^{n}$ defines a facet for $D P(L(C))$,

(ii) $\alpha^{t} x \geq \beta$ with $x \in \mathbb{R}^{n}$ defines a facet for $\operatorname{EDP}(C)$,

(iii) $\alpha^{t} x \leq \sum_{e \in E(C)} \alpha(e)-\beta$ with $x \in \mathbb{R}^{n}$ defines a facet for $S F P(C)$.

Hence, the polytopes $S F P(C), D P(L(C))$ and $\operatorname{EDP}(C)$ are equivalent in the sense that there is a one-to-one correspondence between their facets.

Proof. Note that these polytopes are all defined in $\mathbb{R}^{n}$ and are full dimensional. The lemma follows from the relations described in Lemmas 4.2 and 4.3 as they are all preserved under affine transformations.

As in [8], a complete linear description for $D P(L(C))$ is given, by Lemma 4.4, we can also derive complete descriptions for $S F P(C)$ and $\operatorname{EDP}(C)$. We will explicit these complete descriptions in the following section. 


\subsection{Complete description of $\operatorname{SFP}(C)$}

Let us consider the graph $L(C)$ and the polytope $D P(L(C))$ in $\mathbb{R}^{n}$ whose component indexed by the nodes in $V^{L}$. In [9] and [8], Bouchakour et al. give the following integer formulation for MWDSP,

$$
\begin{array}{ll}
\min c^{t} x & \text { for all } v \in V^{L} \\
0 \leq x_{v} \leq 1 & \text { for all } v \in V^{L} \\
x(N(v)) \geq 1 & \text { for all } v \in V^{L} \\
x_{v} \text { integer } &
\end{array}
$$

They have also characterized two classes of facet-defining inequalities for $D P(L(C))$.

Theorem 4.5. [9] The inequality

$$
x\left(V^{L}\right) \geq\left\lceil\frac{|C|}{3}\right\rceil
$$

defines a facet for $D P(L(C))$ if and only if either $|C|=3$ or $|C| \geq 4$ and $|C|$ is not a multiple of 3 .

Theorem 4.6. [8] Let $W=\left\{v_{1}, \ldots, v_{p}\right\}$ be a subset of $p \geq 3$ nodes in $V^{L}$ satisfying the following conditions:

C1: $p$ is odd and $v_{1}<v_{2}<\ldots<v_{p}$,

C2: $\left|C\left(v_{i}, v_{i+1}\right)\right|=3 k_{i}, k_{i} \geq 1$, for $i=1, \ldots, p$ with $v_{p+1}=v_{1}$.

Then the constraint

$$
2 \sum_{v \in W} x_{v}+\sum_{v \in V^{L} \backslash W} x_{v} \geq \sum_{i=1}^{p} k_{i}+\left\lceil\frac{p}{2}\right\rceil
$$

defines a facet for $D P(L(C))$.

Let us apply Lemma 4.4 to derive facet-definining inequalities for $S F P(C)$. It is clear that applying Lemma 4.4 to inequalities (4.1) yields the trivial inequalities

$$
0 \leq x_{e} \leq 1 \text { for all } e \in E(C)
$$

for $\operatorname{SFP}(C)$.

By applying Lemma 4.4 to inequalities (4.2), we get the 3-path inequalities

$$
x(P) \leq 2 \text { for all path of length } 3 \text { in } C,
$$

for $\operatorname{SFP}(C)$ which have been described in Section 2. The following proposition can be obtained by applying Lemma 4.4 to inequalities (4.3).

Proposition 4.7. The cycle inequality

$$
x(E(C)) \leq\left\lfloor\frac{2|C|}{3}\right\rfloor
$$

defines a facet for $\operatorname{SFP}(C)$ when, either $|C|=3$ or $|C| \geq 4$ and $|C|$ is not multiple of 3 .

Let $W=\left\{v_{1}, \ldots, v_{p}\right\} \subset V^{L}$ be a subset of nodes of $V^{L}$ as defined in Theorem 4.6, let $f_{i}$ denote the edge in $C$ corresponding to the node $v_{i}$ in $L(C)$ and let $M=\left\{f_{1}, \ldots, f_{p}\right\}$. For $i=1, \ldots, p$, we define $C\left(f_{i}, f_{i+1}\right)$ with $f_{p+1}=f_{1}$ to be the path between $f_{i}$ and $f_{i+1}$ in $C$ which does not contain any edge in $M$. The conditions C1 and $\mathrm{C} 2$ on the set $W$ can be transformed into conditions M1 and M2 on the set $M$ as follows:

M1: $M$ is a matching of odd cardinality,

M2: $\left|C\left(f_{i}, f_{i+1}\right)\right|=3 k_{i}, k_{i} \geq 1$, for $i=1, \ldots, p$ with $f_{p+1}=f_{1}$. 
We then deduce the following result by applying Lemma 4.4 to inequalities (4.4).

Proposition 4.8. The matching-cycle inequalities

$$
2 x(M)+x(E(C) \backslash M)+\leq 2 \sum_{i=1}^{p} k_{i}+\left\lfloor\frac{3 p}{2}\right\rfloor \text { for all } M \subset E(C) \text { satisfying conditions M1 and M2. }
$$

define facets for $\operatorname{SFP}(C)$.

Proof. Given a matching $M$ of $C$ satisfying conditions M1 and M2, suppose that $v_{1}, \ldots, v_{p}$ are the nodes in $L(C)$ corresponding respectively to $f_{1}, \ldots, f_{p}$. It is easy to see that $v_{1}, \ldots, v_{p}$ satisfy conditions $\mathrm{C} 1$ and $\mathrm{C} 2$ of Theorem 4.6, and hence, we have that

$$
2 \sum_{v \in W} x_{v}+\sum_{v \in V^{L} \backslash W} x_{v} \geq \sum_{i=1}^{p} k_{i}+\left\lceil\frac{p}{2}\right\rceil
$$

defines a facet for $D P(L(C))$. The result thus follows from Lemma 4.4.

In [8], Bouchakour et al. have shown the following theorem.

Theorem 4.9. [8] A complete linear description for $D P(L(C))$ is given by inequalities (4.1), (4.2), (4.3), (4.4).

As a direct consequence, we have the following result.

Corollary 4.10. When $G$ is a cycle, $\operatorname{SFP}(G)$ is completely described by the trivial inequalities, the 3-path inequalities, the cycle inequality (4.5) and the matching-cycle inequalities (4.6).

\section{Conclusions}

In this paper, we have presented an IP formulation for MWSFP. We have also given two complete linear descriptions for $\operatorname{SFP}(G)$, the star forests polytope for the cases where $G$ is a tree and $G$ is a cycle. An interesting direction for future works would be to exploit these results to derive a complete linear description for $\operatorname{SFP}(G)$ when $G$ is a cactus. Our complete description for $\operatorname{SFP}(G)$ when $G$ is a tree could be helpful to find an exact solution for the MWSFP in general graphs. A star forest is always a subgraph of a spanning tree in $G$ and a complete linear description of the spanning tree polytope is known.

Acknowledgements. We would like to thank one anonymous referee for her/his helpful comments which greatly improved the presentation of the paper.

\section{REFERENCES}

[1] A. Agra, D. Cardoso, O. Cerfeira and E. Rocha, A spanning star forest model for the diversity problem in automobile industry. In: ECCO XVIII, Minsk (2005).

[2] S. Athanassopoulos, I. Caragiannis, C. Kaklamanis and M. Kyropoulou, An improved approximation bound for spanning star forest and color saving. In: MFCS. Springer, Berlin, Heidelberg (2009) 90-101.

[3] M. Baïou and F. Barahona, On the integrality of some facility location polytopes. SIAM J. Discrete Math. 23 (2009) 665-679.

[4] M. Baïou and F. Barahona, Simple extended formulation for the dominating set polytope via facility location. Tech. Rep. RC25325, IBM Research (2012).

[5] M. Baïou and F. Barahona, Algorithms for minimum weighted dominating sets in cycles and cacti. Tech. Rep. RC25488, IBM Research (2014).

[6] M. Baïou and F. Barahona, The dominating set polytope via facility location. Combinatorial Optimization. ISCO 2014. In Vol. 8596 of Lecture Note Computer Sciences (2014) 38-49.

[7] V. Berry, S. Guillemot, F. Nicholas, and C. Paul, On the approximation of computing evolutionary trees. In: Proc. of the Eleventh Annual International Computing and Combinatorics Conference. Springer, Berlin, Heidelberg (2005) 115-123.

[8] M. Bouchakour, T.M. Contenza, C.W. Lee, A.R. Mahjoub, On the dominating set polytope. Eur. J. Combin. 29 (2008) $652-661$. 
[9] M. Bouchakour and A.R. Mahjoub, One-node cutsets and the dominating set polytope. Discrete Math. 165/166 (1997) 101-123.

[10] B.-M. Bui-Xuan, J.A. Telle and M. Vatshelle, Boolean-width of graphs. Theoret. Comput. Sci. 412 (2011) $5187-5204$.

[11] D. Chakrabarty and G. Goel, On the approximability of budgeted allocations and improved lower bounds for submodular welfare maximization and GAP. In: FOCS '08. IEEE 49th Annual IEEE Symposium on Foundations of Computer Science, 1975 (2008) 687-696.

[12] N. Chen, R. Engelberg, C.T. Nguyen, P. Raghavendra, A. Rudra and G. Singh, Improved approximation algorithms for the spanning star forest problem. In: APPROX/RANDOM In Vol. 4627 of Lecture Notes in Computer Science book series (2007) $44-58$.

[13] T.W. Haynes, P.J. Slater and S.T. Hedetniemi, Fundamentals of Domination in Graphs. CRC Press, Boca Raton, FL (1998).

[14] J.He and H. Liang, On variants of the spanning star forest problem. In: Proc. of FAW-AAIM (2011) 70-81.

[15] T. Ito, N. Kakimura, N. Kamiyama, Y. Kobayashi and Y. Okamoto, Minimum-cost b -edge dominating sets on trees. In Vol. 8880 of Lecture Notes Computer Sciences (2014) 195-207.

[16] A.R. Mahjoub, Polytope des absorbants dans une classe de graphes seuil. Annal. Discrete Math. 17 (1983) $443-452$.

[17] C.T. Nguyen, J. Shen, M. Hou, L. Sheng, W. Miller and L. Zhang, Approximating the spanning star forest problem and its applications to genomic sequence alignment. In: Proc. of SODA (2007) 645-654.

[18] V.H. Nguyen, The maximum weight spanning star forest problem on cactus graphs. Discrete Math. Algorithms App. 7 (2015) 1550018 .

[19] A. Saxena, Some results on the dominating set polytope of a cycle. Technical Report CMU (2004).

[20] M. Yannakakis and F. Gavril, Edge dominating sets in graphs. SIAM J. Appl Math. 38 (1980) 364-372. 